\title{
The Causal Probabilistic Relationship Between Economic Development and Democracy: Evidence from Bayesian Networks Analysis
}

Nedra Baklouti", Younes Boujelbene ${ }^{* *}$

\begin{abstract}
The present work analyzes the evolution of thinking that drives political scientists and economists vis-à-vis the relationship between a political regime and development. This article addresses the important question of whether democracy promotes economic development and helps to provide a powerful framework for the economic results of autocracy and democracy. Given that relations between democratic transition and economic growth are fairly complex with uncertain results, we used a probabilistic approach based on Bayesian networks, which is an ideal tool for the probabilistic modeling of uncertainty. In fact, the selected sample includes 11 countries $^{1}$ of the MENA region. We note that Libya has $92.9 \%$ of high average incomes and $7.14 \%$ of low average incomes; therefore the relationship between democracy and GNI is nonlinear and convex. However, predicting the change of the political regime of scenarios shows that the relationship between the level
\end{abstract}

of income and democracy in Bahrain is nonlinear and concave, since its economy is composed of $71.4 \%$ of high income and $28.6 \%$ of higher average incomes. For the remaining countries, we found that whatever the level of democracy, the income levels remain the same.

\section{JEL: A13, C45, F50}

Keywords: Democracy; Economic growth; Bayesian Networks.

\section{Introduction}

A complex picture of the relationship Dbetween economic and political development has emerged from several empirical studies undertaken in the 1990s (Papaioannou and Siourounis, 2008; Rodrik and Wazciarg, 2005; Persson and Tabellini, 2006; Baklouti and Boujelbene, 2015; 2018). The belief that an authoritarian government is better equipped to promote economic growth and development, in countries with low or medium income level, is part of a broad intellectual tradition (Haan and Siermann (1995), Bhagwati, (2002), Drury et

* Corresponding author: Unit of Research in Applied Economics URECA; Faculty of Economics and Management, University of Sfax; Street of Airport, km 4.5, LP 1088, Sfax 3018, Tunisia.

** Faculty of Economics and Management, University of Sfax; Street of Airport, km 4.5, LP 1088, Sfax 3018, Tunisia.

${ }^{1}$ Tunisia, Algeria, Libya, Morocco, Egypt, Kuwait, Arabia Saoudite, Jordan, Bahrain, Lebanon, Oman 


\section{Articles}

al. (2006), Kelsall and Booth (2013); Booth (2012); Peev and Mueller (2012). Some other researchers (Herring et al 2005; Persson and Tabellini, 2009; Thacker, 2011; Rodrik, 1999) advance the thesis that democracy is favorable to economic growth through a better management of economic shocks and ensuring higher wages.

Often, the equations that link the variables assume that there is a linear relationship between the dependent and independent variables, or a linear relationship between parameters. In many cases, the adoption of a linear form is only justified for technical reasons, mainly because it allows for an easier resolution of the system. In fact, many nonlinear relationships have effects with thresholds, discontinuities, etc. However, the
The Causal Probabilistic Relationship Between Economic Development and Democracy

estimating tools and models of identification are usually unsuitable for similar situations. Therefore, it is often preferred to ignore these nonlinearities which seriously distort the real dynamics of systems.

The forecasts of the evolution of the change of the political regime and highlighting its probable consequences are to plan for the long term. It is the same for many other phenomena, the effect of which on the well-being of future generations can be considerable.

Compared to the previous studies (see Table 1), this paper used the Bayesian Network regression based on the structural modeling to study the nexus between democracy and economic growth in the Middle East and North Africa (MENA) region.

Table1: Summary of the existing empirical studies on the democracy and economic growth relationships.

\begin{tabular}{|l|l|l|l|l|}
\hline Study & Countries & Reviewperiods & AppliedMethodologies & Causalityrelationship \\
\hline Yi Che et al. (2013) & $\begin{array}{l}\text { The United States and Columbia } \\
\text { (a comparative study) }\end{array}$ & $1960-2000$ & GMM in system & G $\rightarrow$ D \\
\hline $\begin{array}{l}\text { Paldam and Gundlach } \\
\text { (2012) }\end{array}$ & A cross-country & $1972-2008$ & OLS & G $\rightarrow$ D \\
\hline $\begin{array}{l}\text { Rodrik and Wazciarg } \\
\text { (2005) }\end{array}$ & 154 countries & $1950-2000$ & Fixedeffect & D $\rightarrow G$ \\
\hline $\begin{array}{l}\text { Papaioannou and } \\
\text { Siourounis (2008) }\end{array}$ & 166 countries & $1960-2003$ & Fixedeffect & D G G \\
\hline Feng (2003) & 106 countries & $1975-1995$ & Granger causality test & D $\rightarrow G$ \\
\hline $\begin{array}{l}\text { Persson and Tabellini } \\
\text { (2006) }\end{array}$ & $\begin{array}{l}150 \text { developed and developing } \\
\text { countries }\end{array}$ & $1960-2000$ & FixedEffect & $D \rightarrow G$ \\
\hline $\begin{array}{l}\text { Benedikt Heid et al. } \\
\text { (2012) }\end{array}$ & 150 countries & Periodafterwar & System GMM & $G \rightarrow D$ \\
\hline
\end{tabular}

NB: $G$ and D indicate GDP and democratic index $\rightarrow$ indicates the unidirectional causality

\section{A Bayesian Network is a logistic regression} and an instrument capable of representing a causal dependency model between the stochastic variables (Pearl 2001). It is used to calculate a posteriori the probabilities or the most probable causes and help predict the change of the future political system scenarios to distinguish the results of these actions on the level of income.

\section{Bayesian Networks}

A Bayesian network is a form of probabilistic graphical model (Taroni et al. 2006). In a Bayesian network, the causal relationships between the variables of interest are probabilistic; which implies that the observation of one or more reasons do not 


\section{Articles}

systematically cause the effect or effects that depend on them, but affect only the probability of realization. The particular interest of the Bayesian networks is to formalize the knowledge and in a distributed and flexible way to represent the complexity due to the diversity of indirect causes. The Bayesian network is the combination between probability theory and graph theory. It provides natural tools to address two major problems commonly encountered in artificial intelligence, applied mathematics or engineering: uncertainty and complexity. It plays, in particular, a growing role in the design and analysis of algorithms related to reasoning or learning (Dawid 1992, Becker and Naim, 1999, Jordan 1999). In this paper, we will focus on a particular model of the family of graphical models: Bayesian networks, which use directed acyclic graphs. The role of graphs in probabilistic and statistical models is threefold; (i) they provide an effective way of expressing hypotheses, (ii) provide an economic representation of joint probability functions, and (iii) facilitate the inference from observation.

A Bayesian network $R=(A, B)$ is defined by:

- $A=(X, Y)$, directed acyclic graph whose

$Y$ nodes are associated with a set of random variables $X=\left\{X_{1}, \cdots, X_{n}\right\}$,

- $B=\{P(X i \mid P a(X i))\}$, all the probabilities of each node $\mathrm{Xi}$ conditional on the state of its relatives $\mathrm{Pa}(\mathrm{Xi})$ in $\mathrm{A}$.

- A set of random variables $X=\left\{X_{1}, \cdots, X_{n}\right\}$ associated with the nodes of the graph such that: $P\left(X_{1}, X_{2} \ldots X_{n}\right)=\Pi P\left(X_{i} \mid P a\right.$ $\left(X_{i}\right)$ ) If there is an arc from node $C$ to node $D, C$ is called the parent of $D$. The set of parent nodes of $X_{i}$ is denoted by $\mathrm{Pa}\left(\mathrm{X}_{\mathrm{i}}\right)$.

\subsection{Building a Bayesian network:}

Several steps have to be considered in building a Bayesian network:

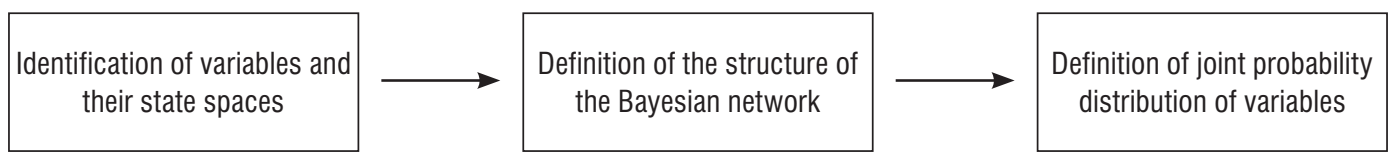

Fig 1. Construction of Bayesian Networks

Source: Naïm et al. 2007

The first step is the identification of variables and all of their possible values. In this step, the intervention of an expert system is always necessary. The second step consists in defining the structure of the Bayesian network and finding influential links between the variables. The last step is the creation of probability tables for the variables for which marginal probabilities must be defined, in this case where conditional probabilities are defined.

\subsection{Treatments with Netica: The naive Bayesian model under Netica}

The Bayesian networks belong to the family of graphical models. The network structure may be described as follows: each node in the graph represents a variable, while the edges between the nodes represent the probabilistic dependencies between the corresponding variables (Ben-Gal, 2007). As part of the naive Bayesian classifier, it is considered that the descriptors are pair-wise conditionally independent from the values of the target variable. Where $\mathrm{X}=$ (democracy, human capital, labor capital, physical capital, corruption, country) is the set of the descriptor variables, and $Y=$ (GNI measures the income level) is the predictor variable. The Netica 5.18 software was used for modeling. The KullbackLeibler was used to assess the degree of dependence between two network variables. A naive Bayesian classifier is a type of a linear classifier that can instead be defined as a simplification of Bayesian networks. Its 


\section{Articles}

structure is in fact composed of only two levels: first with only one node, noted for example $Y$ and the second having multiple nodes for a single parent $Y$. These models are called naive because they make the assumption that all the sons are independent from one another. $Y$ is the son of noted $X_{1} \ldots X n$, the joint probability distribution of a Bayesian classifier is written $P$ $\left(\mathrm{Y} \mid \mathrm{X}_{1} \ldots \mathrm{n}\right) \ldots$

\section{Bayesian Network Method}

The Bayesian networks belong to the family of graphical models. The network structure may be described as follows: each node in the graph represents a variable, while the edges between the nodes represent the probabilistic dependencies between the corresponding variables. Netica ${ }^{\circledR} 5.18$ software (Norsys Corporation, https://www. nursys.com) was used for modeling.

\subsection{Defining network variables and their modalities:}

Many real-world problems involve continuous quantities: height, temperature, currency, weight. In fact, most of the statistical processes as random variables have continuous domains. Continuous variables can assume, by definition, an infinite number of values therefore it is impossible to explicitly specify the conditional probabilities for each of these values. One solution is to avoid continuous variables by discretization, i.e. by dividing the possible values into a set of fixed intervals. In this order we present the variables in the table below:

Table 1. The network variables and their values

\begin{tabular}{|l|l|}
\hline \multicolumn{1}{|c|}{ Variables } & \multicolumn{1}{c|}{ Type } \\
\hline Gross National Income & Discrete $:[1 ; 2 ; 3]$ \\
\hline Democracy(Dem) & Discrete $:[0 ; 1 ; 2]$ \\
\hline Human Capital(H) & Discrete : $[1 ; 2 ; 3 ; 4]$ \\
\hline Physical Capital(K) & Discrete $:[0 ; 1]$ \\
\hline Labour Capital(L) & Discrete : $[0 ; 1]$ \\
\hline Corruption(IPC) & Discrete $:[0 ; 1]$ \\
\hline
\end{tabular}

The Causal Probabilistic Relationship Between Economic Development and Democracy

\subsubsection{Parent Node: Gross National Income}

The economies were divided according to the gross national revenue (GNI) per capita in 2008 and calculated using the Atlas method of the World Bank. The groups are:

- Low-income US \$ 975 at least

- Lower middle income, from US \$9763855

- Middle income, US \$ 3856-11905

- higher income, higher than 11906 US dollars

To achieve quantification, we have to justify both the number of classes and class terminals, therefore I clipped the GNI variable into three classes:

Named first class (S1): [975; $3855[\rightarrow$ Below Average Income

Named 2nd Class (S2): [3856; $11905[\rightarrow$ Superior Middle Income

Named 3rd Class (S3): > $11906 \rightarrow$ High income

\subsubsection{Child Node :}

\section{$\checkmark$ Democracy}

According to Freedom House database, if the variable is democracy:

- Between [1; $2.5[\rightarrow$ Free Country $\rightarrow$ named (SO)

- Between [3; 5] $\rightarrow$ Partly Free Country $\rightarrow$ named (S1)

- Between $[5,5 ; 7] \rightarrow$ not free country $\rightarrow$ named (S2)

\section{$\checkmark$ Corruption}

Corruption is measured by the perception of its index according to Transparency International data base. The extent of corruption is indicated on a scale that ranges from [0 to 10]: the closer it is to 10 , the less the country is corrupted, and the closer it is to 0 , the more corrupted the country is. Hence, the discretization of the index of perception of corruption is as follows: 


\section{Articles}

- Between [0 ; 5[ $\rightarrow$ corrupted country $\rightarrow$ named (SO)

- Between] 5 ; 10] $\rightarrow$ uncorrupted country $\rightarrow$ named (S1)

\section{$\checkmark$ Human Capital}

Human capital is approximately measured by registration in high schools (Gross) extracted from the World Bank database. The gross enrollment ratio can exceed $100 \%$ due to the inclusion of students aged over or under the age following early or late entry and failure. Therefore, the discretization of this variable is as follows:

- If the rate is between $[0 ; 25[\rightarrow$ named (S1)

- If the rate is between [25; $50[\rightarrow$ named (S2)

- If the rate is between $[50 ; 75[\rightarrow$ named (S3)

- If the rate is between [75; >100[ $\rightarrow$ named (S4)

\section{$\checkmark$ Physical Capital}

Physical capital is approximately measured by gross capital formation (\% GDP) extracted from the World Bank database. The discretization of this variable is as follows:

- Between [0;25[ $\rightarrow$ named (SO)

- Between [25;50[ $\rightarrow$ named (S1)

\section{$\checkmark$ Labour Capital}

Human capital is approximately measured by the rate of participation in the total active population (\% of total population aged 15 and over), all individuals who supply labor for the production of goods and services during a given period. The discretization of this rate is as follows:

- If the rate is $<50 \% \rightarrow$ named SO

- If the rate is $>50 \% \rightarrow$ named S1

\section{Learning Bayesian Networks from data}

The probability tables that are provided by the software Netica are presented below:

Table 2. Probability table for Medium-low income countries

\begin{tabular}{|c|c|c|c|c|c|c|c|c|c|c|c|c|}
\hline \multirow{5}{*}{$\begin{array}{l}\text { Tunisia } \\
\text { Morocco } \\
\text { Algeria } \\
\text { Egypt } \\
\text { Jordan }\end{array}$} & \multicolumn{2}{|c|}{ Democracy } & \multicolumn{2}{|c|}{ K } & \multicolumn{2}{|r|}{$\mathbf{L}$} & \multicolumn{2}{|r|}{ H } & \multicolumn{2}{|r|}{ CPI } & \multicolumn{2}{|c|}{ GNI } \\
\hline & So & $0 \%$ & S0 & $46,5 \%$ & So & $83,1 \%$ & S1 & $0 \%$ & So & $88,7 \%$ & S1 & $100 \%$ \\
\hline & S1 & $36,6 \%$ & S1 & $535 \%$ & S1 & $160 \%$ & S2 & $11,3 \%$ & S1 & $11,3 \%$ & S2 & $0 \%$ \\
\hline & S2 & $63,4 \%$ & 01 & $30, \mathrm{~J} \%$ & 31 & $10,9 \%$ & S3 & $22,5 \%$ & & & S3 & $0 \%$ \\
\hline & & & & & & & S4 & $66,2 \%$ & & & & \\
\hline
\end{tabular}

Table 2 presents the descriptive analysis of lower middle income countries (Fig.8), such as Tunisia, Morocco, Algeria and Egypt, where who's GNI belong to the low-income as already shown above. Since the variable democracy is the index of democracy established by Freedom House by taking the average of the political rights and civil liberties, it is rescaled so that the value is ranked from 1 (most democratic) to 7 (least democratic). $36.6 \%$ of the citizens are partially free and $63.4 \%$ are not free and do not enjoy civil and political rights. In these five countries, $46.5 \%$ of the investments are strictly below 25\% of the GDP and the rest, i.e. $53.5 \%$ represent at least $25 \%$ and can reach up to $49 \%$ of the GDP of these countries. Regarding the corruption perception Index (CPI), which indicates the perceived level of corruption in public administration and political class in the country, we found that $88.7 \%$ of bureaucrats and politicians are corrupt and $11.3 \%$ are not. Regarding the human capital, we found that $66.2 \%$ of the population of the countries listed above belong to the fourth skewer, that is to say, human capital is highly qualified. A very recent study by the OECD has helped to clarify 


\section{Articles}

this issue and has shown that in the member countries, an additional year of studying leads to the increase of production per capita by 4-7 percent in the medium and long term. Finally,
The Causal Probabilistic Relationship Between Economic Development and Democracy

we found that $83.1 \%$ of the populations of these countries have a participation rate in the workforce with less than $50 \%$. This result is due to the aging of the workforce in these countries.

Table 3. Probability table for Medium-higher income countries

\begin{tabular}{|c|c|c|c|c|c|c|c|c|c|c|c|c|}
\hline \multirow{4}{*}{$\begin{array}{l}\text { Saoudite } \\
\text { Lebanon } \\
\text { Oman }\end{array}$} & \multicolumn{2}{|c|}{ Democracy } & \multicolumn{2}{|c|}{$K$} & \multicolumn{2}{|c|}{ L } & \multicolumn{2}{|c|}{ H } & \multicolumn{2}{|c|}{ CPI } & \multicolumn{2}{|c|}{ GNI } \\
\hline & SO & $0 \%$ & SO & $64,4 \%$ & SO & $37,3 \%$ & S1 & $0 \%$ & SO & $74,6 \%$ & $\mathrm{~S} 1$ & $0 \%$ \\
\hline & S1 & $11,9 \%$ & & 2560 & c1 & & S2 & $0 \%$ & C1 & & S2 & $100 \%$ \\
\hline & S2 & $88,1 \%$ & S1 & $35,6 \%$ & S1 & b2, $1 \%$ & S3 & $5,08 \%$ & S1 & $25,4 \%$ & S3 & $0 \%$ \\
\hline & & & & & & & S4 & $94,9 \%$ & & & & \\
\hline
\end{tabular}

Table 3 presents the descriptive analysis of upper middle income countries (Fig.8) such as Saudi Arabia, Lebanon and Oman where the GNI belong to the second class as already shown above. In these countries, $11.9 \%$ of the citizens are partly free and $88.1 \%$ are not. For these three countries, $64.4 \%$ of investments are strictly below $25 \%$ of the GDP and the rest i.e.; $35.6 \%$ represent at least
$25 \%$ and can reach up to $49 \%$ of the GDP of these countries. $74.6 \%$ of bureaucrats and politicians are corrupt and the complement to $100 \%$ results from the uncorrupted. $94.9 \%$ of the population is educated, with a gross enrollment rate higher than $75 \%$. Conversely, in low income, the majority of the population is active since these countries are considered young.

Table 4. Probability table for High-income countries

\begin{tabular}{|c|c|c|c|c|c|c|c|c|c|c|c|c|}
\hline \multirow{4}{*}{ Kuwait } & \multicolumn{2}{|c|}{ Democracy } & \multicolumn{2}{|c|}{ K } & \multicolumn{2}{|c|}{ L } & \multicolumn{2}{|c|}{ H } & \multicolumn{2}{|c|}{ CPI } & \multicolumn{2}{|c|}{ GNI } \\
\hline & SO & $0 \%$ & So & $91,7 \%$ & So & $0 \%$ & S1 & $0 \%$ & So & $58,3 \%$ & S1 & $0 \%$ \\
\hline & S1 & $83,3 \%$ & S1 & 8330 & S1 & $100 \%$ & S2 & $0 \%$ & S1 & $417 \%$ & S2 & $0 \%$ \\
\hline & S2 & $16,7 \%$ & | & $0,35 \%$ & | & $100 \%$ & S3 & $0 \%$ & | & $41, \Gamma \%$ & S3 & $100 \%$ \\
\hline & & & & & & & S4 & $100 \%$ & & & & \\
\hline
\end{tabular}

Table 4 shows the descriptive analysis of Kuwait that has a higher average income and whose GNI belongs to the third class as already shown above. Kuwait is the only country that reached $100 \%$ for both human capital and labor. This perspective implies that the labor market is able to absorb many more people in the coming decades.

For the second box (partly free) of the variable of democracy we find a probability of $83.3 \%$. However, if we compare the Kuwaiti political model to that of the other Gulf monarchies, it is clear that none of them has reached the political level of openness currently enjoyed by Kuwait, despite the reforms conducted in this region of the Arab world since the 1990s. However, $58.3 \%$ of the civil servants and politicians are corrupt and $41.7 \%$ are not.

Table 5. Probability table for Libya

\begin{tabular}{|c|c|c|c|c|c|c|c|c|c|c|c|c|}
\hline \multirow{4}{*}{ Libya } & \multicolumn{2}{|c|}{ Democracy } & \multicolumn{2}{|c|}{ K } & \multicolumn{2}{|c|}{$\mathbf{L}$} & \multicolumn{2}{|c|}{ H } & \multicolumn{2}{|c|}{ CPI } & \multicolumn{2}{|c|}{ GNI } \\
\hline & So & $0 \%$ & SO & $63,1 \%$ & So & $40,6 \%$ & S1 & $0 \%$ & SO & $75,9 \%$ & S1 & $7,14 \%$ \\
\hline & S1 & $13,6 \%$ & & & & & S2 & $0,80 \%$ & S1 & 24. & S2 & $92,9 \%$ \\
\hline & S2 & $86,4 \%$ & S1 & $36,9 \%$ & S1 & $59,4 \%$ & S3 & $6,33 \%$ & SI & $24,4 \%$ & S3 & $0 \%$ \\
\hline & & & & & & & S4 & $92,9 \%$ & & & & \\
\hline
\end{tabular}




\section{Articles}

Tables 5 and 6 show the probabilities of the variables for Libya and Bahrain. For the Libyans (Fig.9), the revenues are divided between the 1st and 2 nd class with a rate of $7.14 \%$ for the first and $92.9 \%$ for the second, that is to say Libya may be integrated into the group of countries with higher average income. However, for the other variables, the probabilities are almost the same. Bahrain's economy is heavily dependent on oil, which

represents $60 \%$ of its exports, $70 \%$ of its government revenues and $30 \%$ of its GDP. Bahrain is the third country in the Persian Gulf that began to drill for oil in 1932 (after Iran and Iraq). In addition, this country has a wide economic freedom and a greater legal stability, the Index of Economic Freedom in 2013 ranked the country 13th in economic freedom.

Table 6. Probability table for Bahrain

\begin{tabular}{|c|c|c|c|c|c|c|c|c|c|c|c|c|}
\hline \multirow{4}{*}{ Bahrain } & \multicolumn{2}{|c|}{ Democracy } & \multicolumn{2}{|c|}{ K } & \multicolumn{2}{|c|}{ L } & \multicolumn{2}{|c|}{ H } & \multicolumn{2}{|c|}{ CPI } & \multicolumn{2}{|c|}{ GNI } \\
\hline & S0 & $0 \%$ & so & $83,9 \%$ & so & $10,7 \%$ & S1 & $0 \%$ & SO & $63 \%$ & S1 & $0 \%$ \\
\hline & S1 & $62,9 \%$ & & & & & S2 & $0 \%$ & & & S2 & $28,6 \%$ \\
\hline & \begin{tabular}{|l|} 
S2 \\
\end{tabular} & $37,1 \%$ & S1 & $16,1 \%$ & S1 & $89,3 \%$ & S3 & $1,45 \%$ & S1 & $3 / \%$ & S3 & $71,4 \%$ \\
\hline & & & & & & & S4 & $98,5 \%$ & & & & \\
\hline
\end{tabular}

Table 6 shows, that the revenues of Bahrain (Fig.10) are divided between the 2nd class and 3rd class, with a rate of $28.6 \%$ for the second level and $71.4 \%$ for the third level. Then, we grouped Bahrain and Kuwait together into a high income group.

\section{Results and probabilistic interpretation}

With the specifications presented in the previous section, six Bayesian networks were built using the subsequent variables: democracy, corruption, human capital, physical capital, labor capital, Gross National Income. These variables are continuous and have been composed at intervals based on predefined criteria for database to which they were extracted.

Based on the results of previous work, the overall relationship between growth and democracy is far from being perfect (Efendic et al. 2011). For example, a number of undemocratic countries have significant positive residuals (Kelsall and Booth 2013; Booth 2012). Similarly, countries with an intermediate level of democracy seem to avoid low growth rates, but without reaching particularly high rates. We can only suggest the existence of a nonlinear relationship in which more democracy increases growth when political freedom is weak, but discourages growth when the average level of political freedom is already established. We can only conclude from this evidence that democracy is more or less a key element for economic growth (Barro, 2000).

Scenario 1: Regime change scenario for countries with medium-low income

Fig. 2 shows that vis-à-vis the savings with lower middle income, whatever the nature of the political regime, the level of growth and income remains unchanged. In other words, in countries such as Tunisia, Morocco, Algeria, Egypt and Jordan, whether they are considered free (S0), partially free (S1) or not free (S2), the gross national income still varies between 975 dollars and 3855 dollars. The results for each hypothesis are given in percentage in Fig. 3 and Fig. 4 showing that the conditional probabilities are unchanged for economies with higher average income, no matter what the political regime category is. 

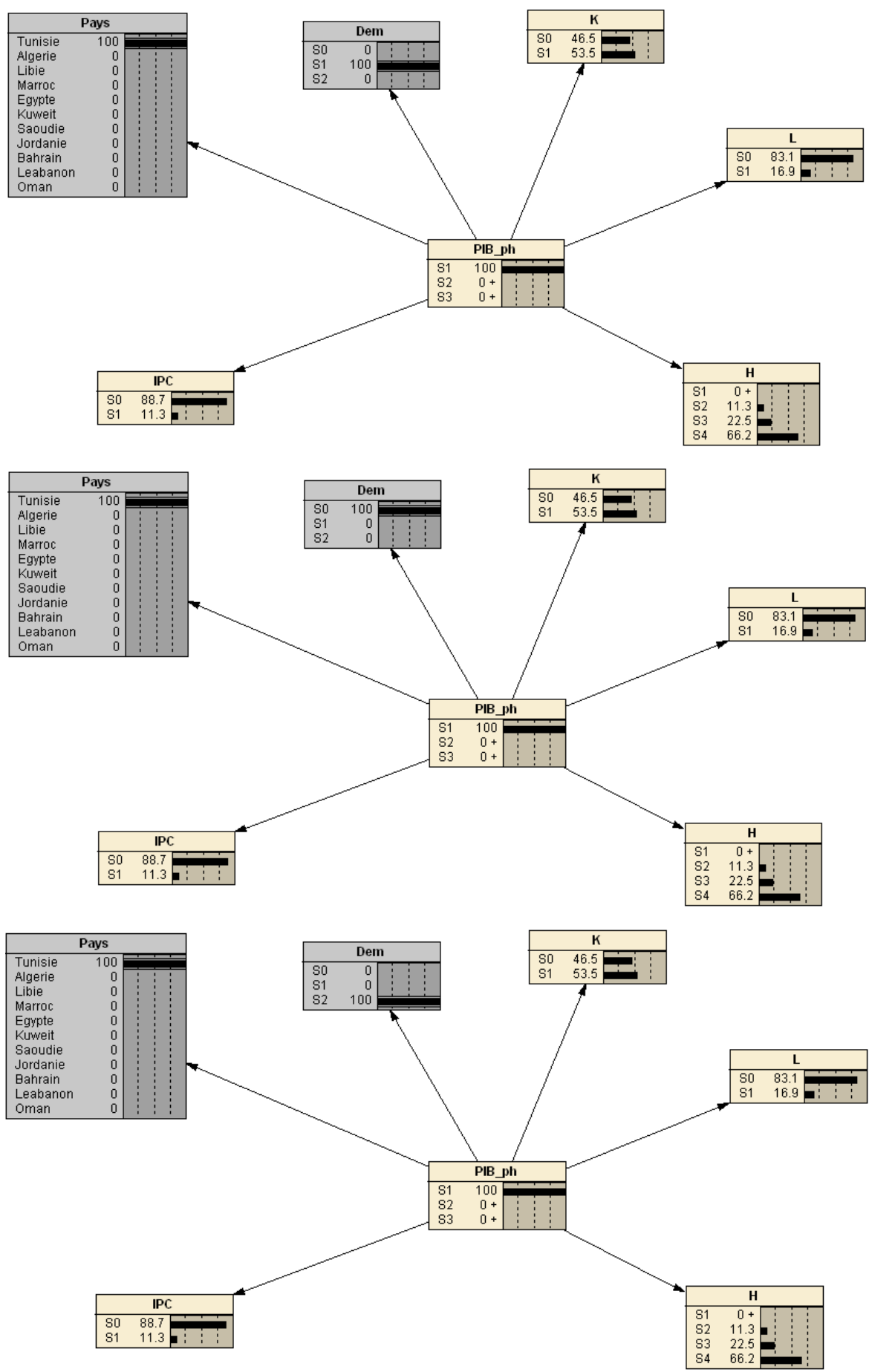

Fig 2. The results of the prediction for the politico-economic scenario for Medium-low income countries. 


\section{Articles}
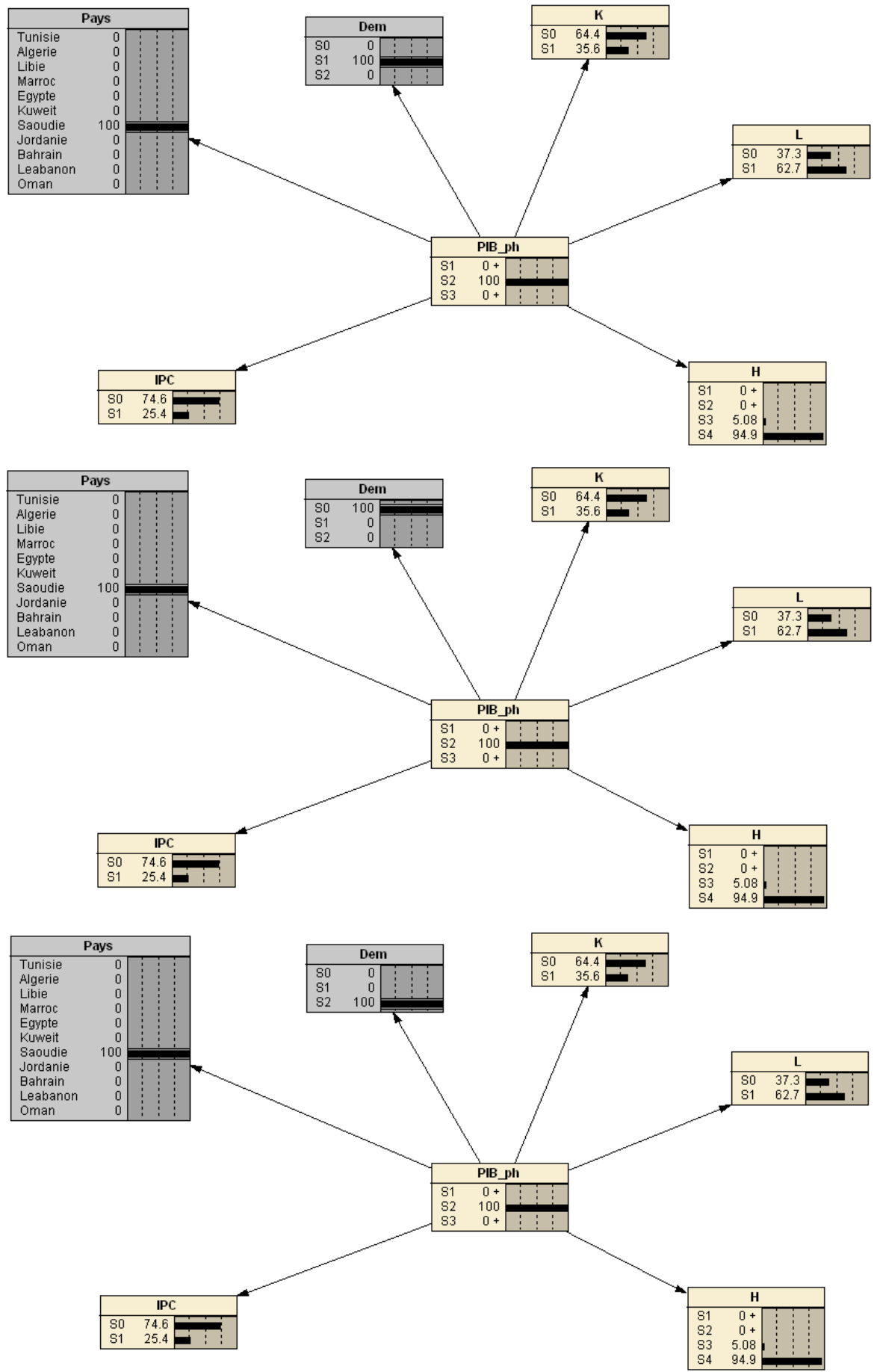

Fig 3. The results of the prediction for the politico-economic scenario for Medium-higher income countries. 

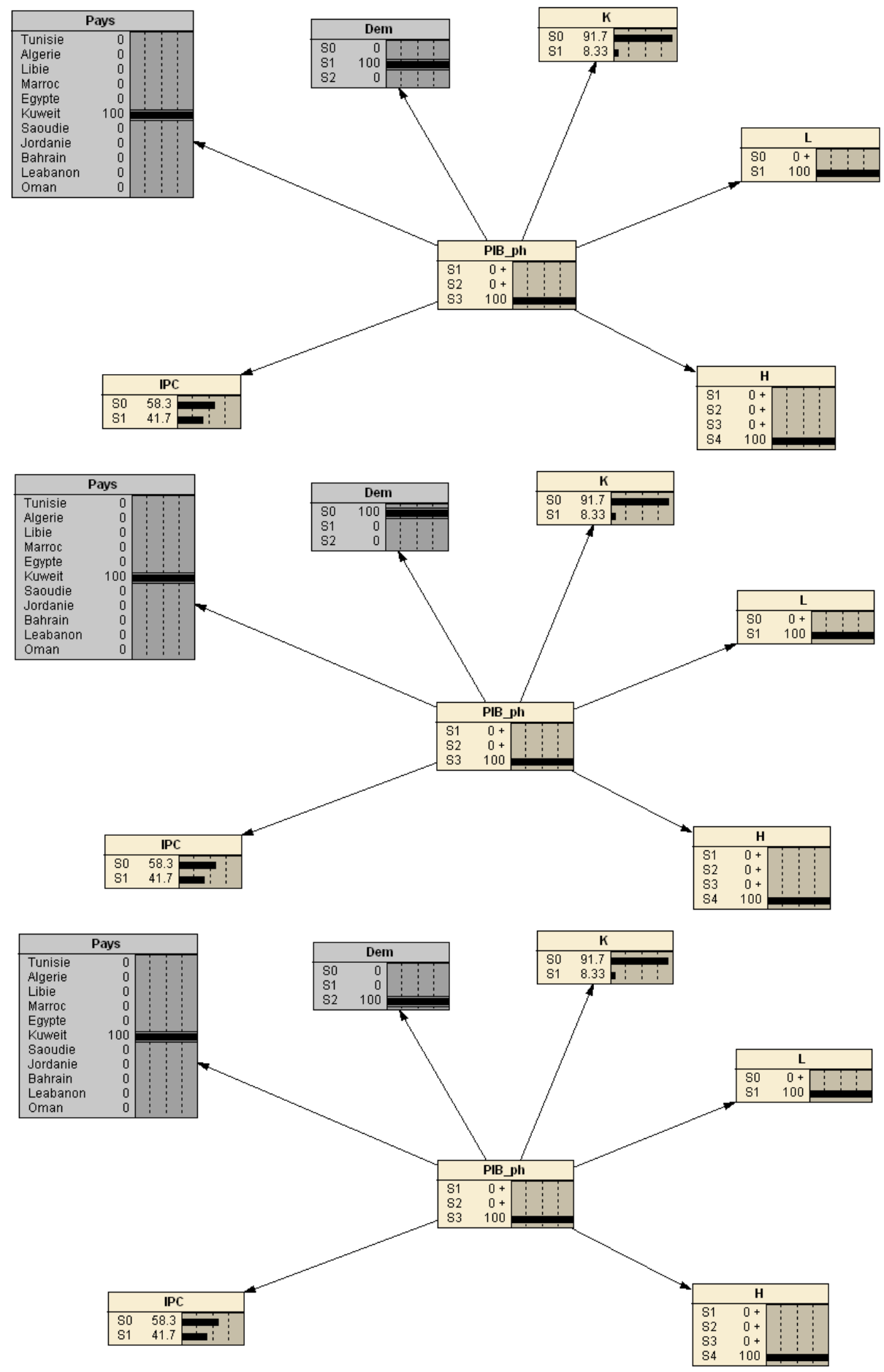

Fig 4. The results of the prediction for the politico-economic scenario for High-income countries. 


\section{Articles}

Scenario 2: Regime change scenario for Libya

Fig. 5 shows that the conditional probabilities of GNI vary with the change in the hypothesis of the nature of the political regime for Libya. With the assumption that the country is free (SO), we found that $94 \%$ of incomes vary between 3856 dollars and 11,905 dollars, but $6.01 \%$ of revenues range between 975 and 3855 dollars. If the country is $100 \%$ partly free (S1), $80.8 \%$ of its revenues are in 2nd class (S2) and 19.2\% in the 1st class (S1). However, the hypothesis of non-free (S2) gives almost the same results as the hypothesis of free countries (S0). We can conclude that the relationship between democracy and the income level is $\mathrm{U}$-shaped, so that it is a convex relationship. Conversely, Barro (1996) highlights the existence of a concave relationship between democracy and economic growth. Moreover, his analysis suggests the presence of a nonlinear relationship: democracy stimulates growth for low levels of political freedom, but tends to reduce it when a certain level of freedom is achieved. The improvement of living standards, whether measured by the increase in GDP, by life expectancy and by education, increases the probability that a country adopts a democratic regime. Barro (1996) adds that this is a peak below which the relationship between democracy and economic growth is positive, but above this point, this relationship is reversed. In other words, democracy would increase economic growth for countries with a low level of political freedom; however, it tends to decrease it when a certain degree of freedom is achieved. The part between the starting point and the peak point having an upward slope is defined by Kruzman et. al (2002) as the "winwin". In our case, the descending part reflects that it is a free country, the lower their income level is until it reaches the partly free regime (the curve reaches its minimum). Then, after this minimum, the curve returns to its growth. In fact, maintaining a more or less authoritarian austere practice is considered essential for the preservation of strong economic growth for the country to benefit from greater prosperity and greater stability.

This result corroborates those of Haan and Siermann (1995), Bhagwati (2002), Drury et al. (2006), Peev and Mueller (2012), Kelsall and Booth (2013), Booth (2012) and Jamshidi (2014), which show that non-democratic countries can also achieve economic growth.

However, other studies suggest there is a beneficial effect of democracy on growth (Thacker, 2011; Hellmanzik, 2013; Sandalicar, 2013; Acemoglu et al 2014), whereas others found no effect of the first on the second (Przeworski and Limongi, 2000; Acemoglu et al. 2008; Yang, 2008).

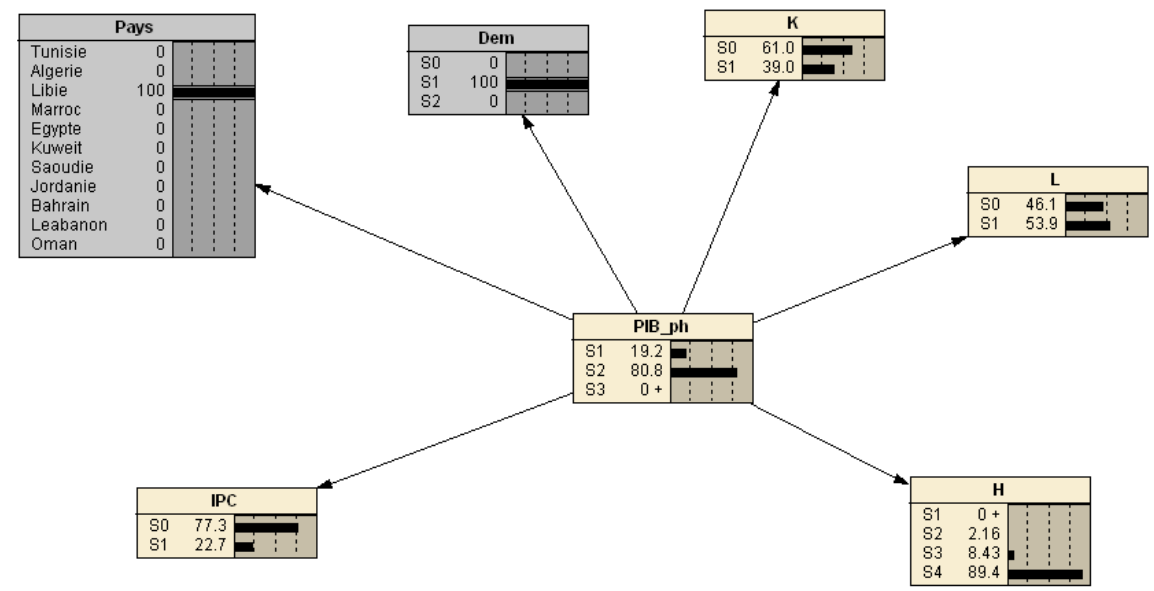



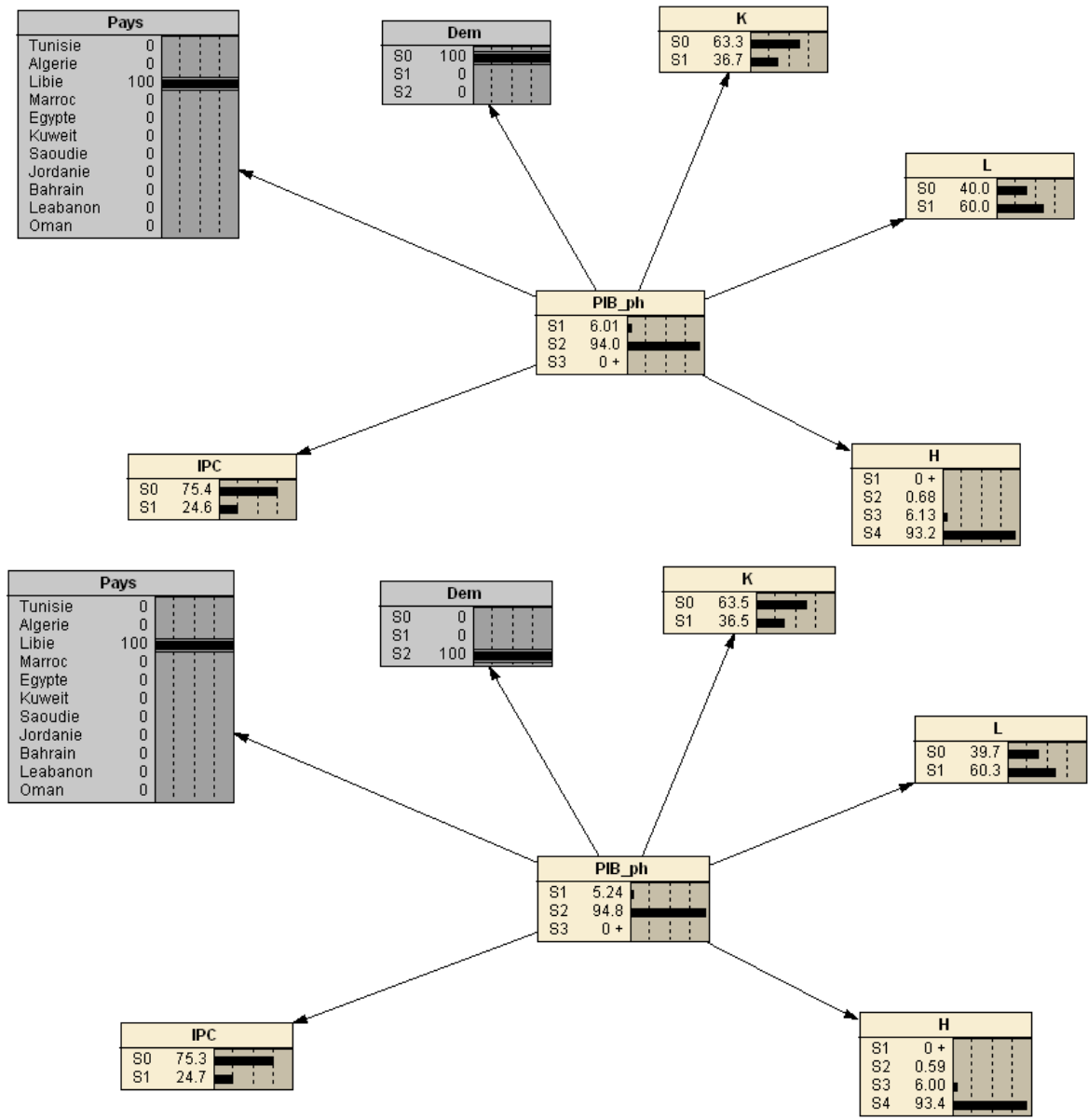

Fig 5. The results of the prediction for the politico-economic scenario for Libya.

Scenario 3: Regime change scenario for Bahrain

Bahrain is classified as a country with high income levels like Kuwait. Fig. 7 shows that the conditional probabilities of GNI vary with the change of the hypothesis of the nature of the political regime for that country.For example: the assumption of freedom of civil and political rights at a rate of $100 \%$ (SO); $86 \%$ of incomes over 11,905 American dollars and $14 \%$ of income between 3856 and 11905 dollars. If we accept the hypothesis of a partly free political regime (S1), we find that the GNI rises to $94.6 \%$ of high income (S3) and $5.39 \%$ of high average income (S2). However, with the hypothesis non-free (S2), the conditional probabilities are reversed, so that $67.9 \%$ of the revenues belong to the 2nd class and only $32.1 \%$ to the 3rd class. We therefore accept that the relationship between democracy and the level of income for Bahrain has a $U$ inverted curve, so it is a concave relationship.

This result is in line with that of Barro (1996) who argued that democracy can be beneficial for the development of countries that are not very democratic, but may delay growth for countries that have already achieved a substantial amount of democracy. This nonlinear relationship is represented in Fig.6. 
Articles

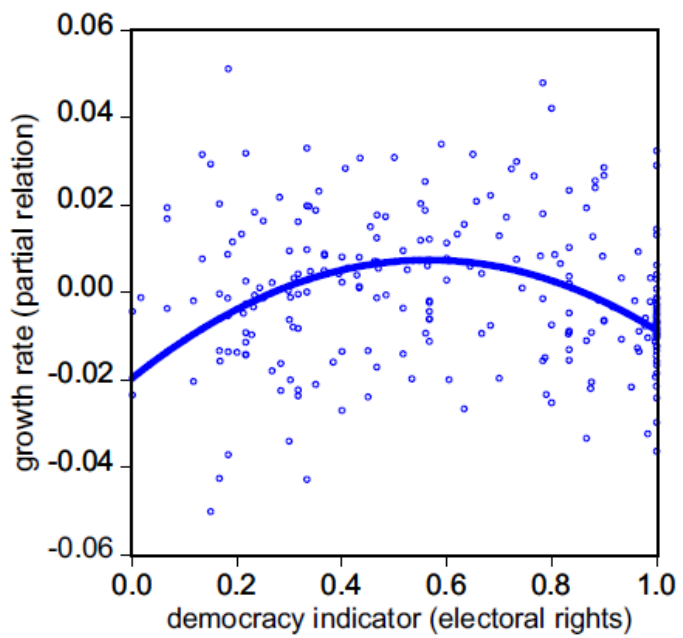

Fig 6. Growth Rate versus Democracy (partial relation) Source: Barro (2003)
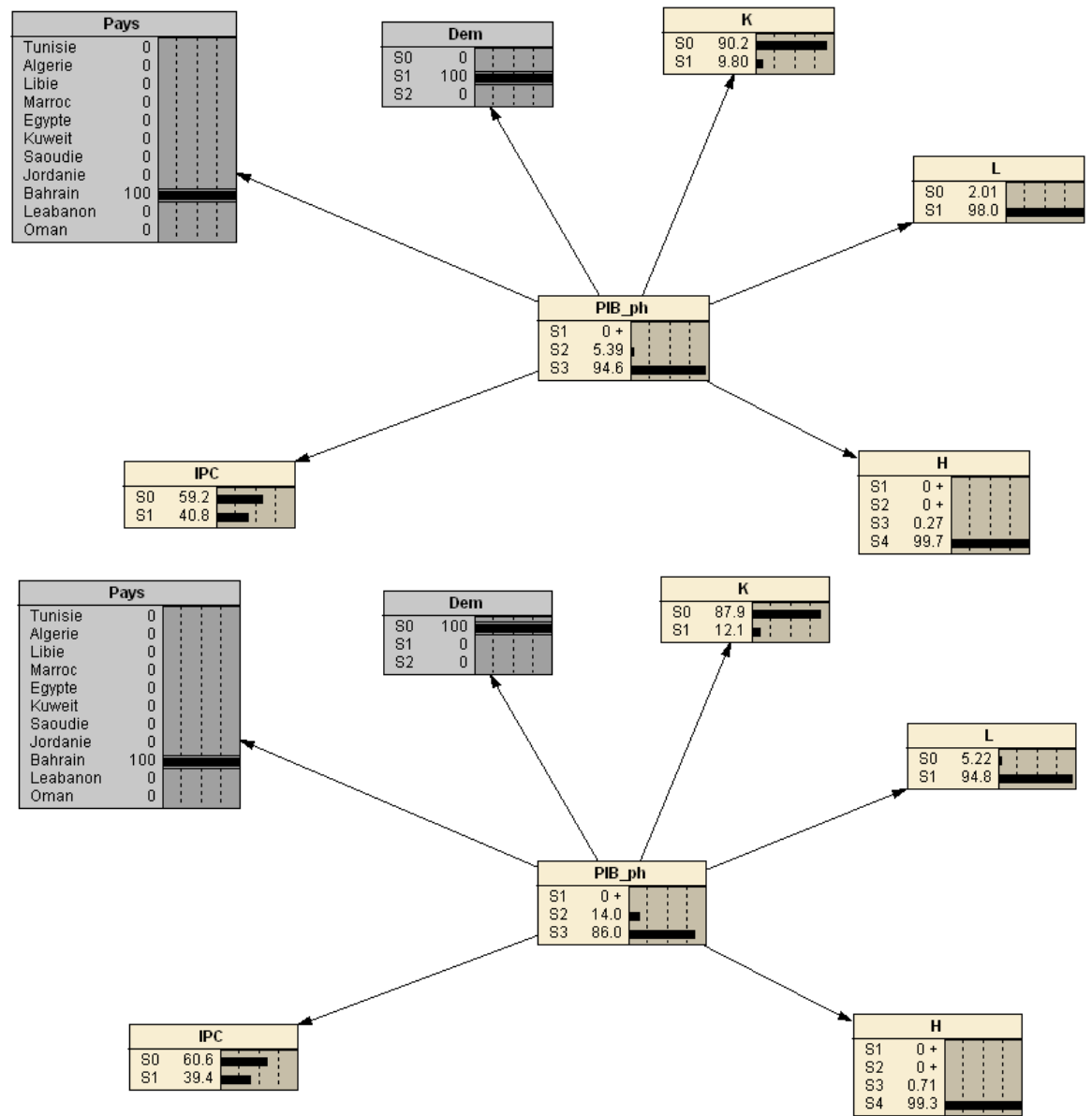


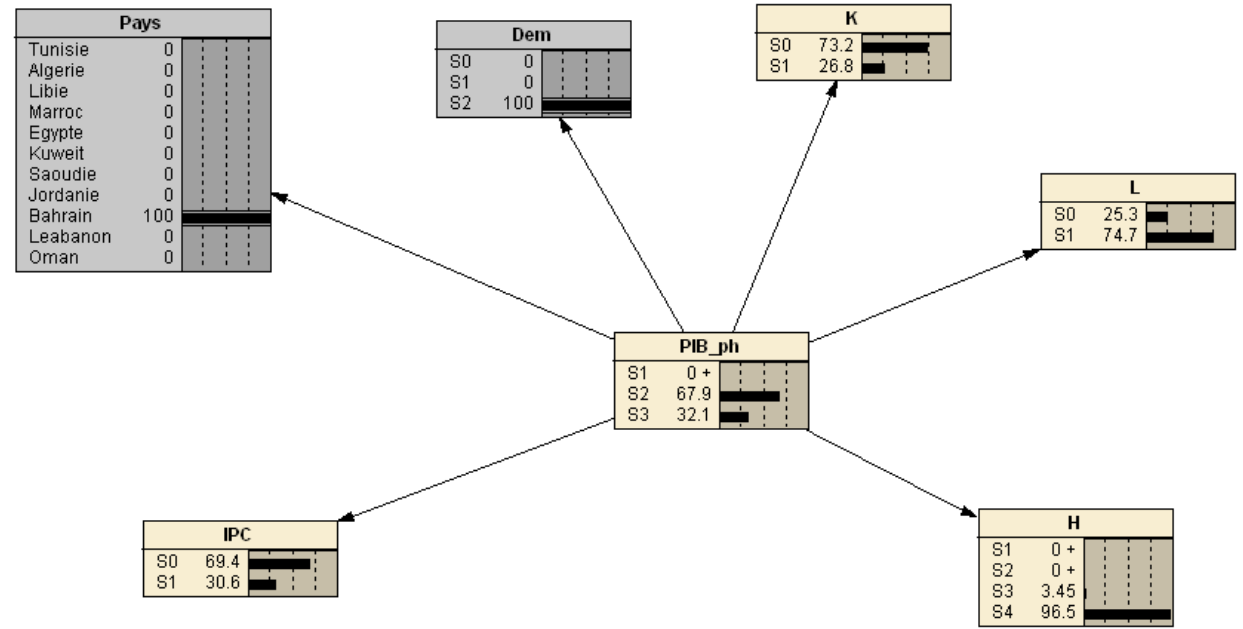

Fig 7. The results of the prediction for the politico-economic scenario for Bahrain.

\section{Conclusion}

This study demonstrated the interest of indicators based modeling using the naive Bayesian networks to analyze the political and economic relations in 11 countries in the MENA region. This modeling embraces a broad set of contemporary political and economic issues by estimating the effects of democratization on the path of economic growth in countries that are either "partly free" or "not free".

Through scenarios predicting, Bayesian networks can integrate the complexity of the study area and the uncertainty of possible relationships, to achieve a causal probabilistic model.The results of the exploratory phase of the scenarios, which defines a development strategy, that is to say, to design polical actions required to achieve the objectives, show that (i) the relationship between democracy and the level of income is dependent on the different levels of wealth between countries; (ii) the relationship is not linear; (iii) the transition to democracy for Bahrain can bring substantial growth benefits; (iv) democracy can hinder economic growth for Libya; (V) the existence of a systemic relationship between democracy and growth for Tunisia, Morocco, Algeria and Egypt as countries with lower average income, Saudi Arabia, Lebanon and Oman as upper middle income countries and Kuwait as a high-income country. The different scenarios regarding the relationship between the political systems, in terms of growth performance, highlight the existence of a rich government as a necessary condition for sustainable growth in the long term.

Our study may be of great help to the Bahrain public autorities to select the most appropriate tools to conduct assessments in terms of civil liberties and political rights of their plans, programs and strategies. The public authorities of other countries in the sample may act on other variables such as human capital and educational level to promote growth and accelerate the catchingup with the developed countries. 


\section{Articles}

\section{References}

Acemoglu, D., Johnson, S., Robinson, J.A., Yared, P., (2008). Income and Democracy, American Economic Review, 98 (3), 808-842.

Acemoglu, D., Naidu, S., Restrepo, P., \& Robinson, J. A. (2014). Democracy does cause growth (No. w20004). National Bureau of Economic Research.

Baklouti, N., \&Boujelbene, Y. (2015). Exploring the Relationship between Democracy, Corruption and Economic Growth in MENA countries. ActaUniversitatisDanubius. CEconomica, 11 (3).

Baklouti, N., \&Boujelbene, Y. (2018). The nexus between democracy and economic growth: evidence from dynamic simultaneousequations models. Journal of the Knowledge Economy, 9(3), 980-998.

Barro, R. J. (1996). Democracy and growth. Journal of Economic Growth, 1, 1-27.

Barro, R., (2000). Inequality and Growth in a Panel of Countries. Journal of Economic Growth 5, 5-32.

Barro, R. J. (2003). Determinants of economic growth in a panel of countries.Annals of economics and finance, 4, 231-274.

Becker, A. Naïm, P. (1999). Les réseaux bayésiens. Eyrolles, eyrolles edition.

Bhagwati, J.N. (2002). Democracy and development: cruel dilemma or symbiotic relationship? Review of Development Economics, 6(2), 151-162.

Dawid, A. P. (1992). Applications of a general propagation algorithm for probabilistic expert systems. Statistics and Computing 2, 25-36.

Drury, A. C., Krieckhaus, J., Lusztig M. (2006). Corruption, democracy and economic growth. International Political Science Review, 27(2), 121-136.
Efendic, A., Pugh, G., Adnett, K. (2011). Institutions and economic performance: A meta-regression analysis. European Journal of Political Economy, 27 (2011) 586-599.

Haan, J.D., Siermann, C.L.J. (1995).New evidence on the relationship between democracy and economic growth. Public Choice, 86, 175-198.

Jordan, M. I., editor (1999). Learning in Graphical Models. MIT Press.

Jordan, M. I., Ghahramani, Z., Jaakkola, T., Saul, L. K. (1999). An introduction to variational methods for graphical models. Machine Learning, 37(2): 183-233.

Kelsall, T., D. Booth (2013). Business, Politics, and the State in Africa: Challenging the Orthodoxies on Growth and Transformation. London: Zed Books.

Kruzmane D., Jankevica L., levinsh G. (2002). Effect of regurgitant from Leptinotarsadecemlineata on wound responses in Solanum tuberosum and Phaseolus vulgaris plants. Physiol. Plant. 115: 577-584.

Papaioannou, Elias, GregoriosSiourounis. (2008). Democratisation and Growth. The Economic Journal, 118: 1520-51.

Pearl, J. (2001). Causality - Models, reasoning and inference. Cambrige University Press.

Peev, E. et Mueller, D.C. (2012). "Democracy, Economic Freedom and Growth in Transition Economies". Kyklos, 65 (3): 371-407.

Persson, Torsten, Guido Tabellini (2006) "Democracy and Development: The Devil in the Details", American Economic Review Papers and Proceedings, 99(2): 319-314.

Persson, Torsten, Guido Tabellini. (2009). "Democratic Capital: The Nexus of Political and Economic Change." American Economic Journal: Macroeconomics, 1(2): 88-126. 
Przeworski, A., M. Alvarez, J. A. Cheibub, F. Limongi, (2000). Democracy and Development, New York: Cambridge University Press.

Rodrik, Dani, and Romain Wacziarg. "Do democratic transitions produce bad economic outcomes?." American Economic Review 95.2 (2005): 50-55.

Rodrik, Dani. (1999). The New Global Economy and Developing Countries: Making Openness
Work. Baltimore: The Johns Hopkins University Press.

Taroni F., Aitken C., Garbolino P., Biedermann A., (2006). Bayesian networks and probabilistic inference in forensic science, Wiley, 2006.

Taroni., F C.G.G. Aitken, P. Garbolino, and A. Biedermann.(2006). Bayesian Networks and Probabilistic Inference in Forensic Science. John Wiley \& Sons, Chichester. 